BMJ Open

Sport \&

Exercise

Medicine

\title{
Assessment and classification of peripheral pain in athletes: a scoping review protocol
}

\author{
Ciarán Purcell (1) , ${ }^{1,2}$ Ciara Duignan, ${ }^{1,2}$ Brona Fullen, ${ }^{1}$ Brian Caulfield ${ }^{1,2}$
}

To cite: Purcell C, Duignan C, Fullen B, et al. Assessment and classification of peripheral pain in athletes: a scoping review protocol. BMJ Open Sport \& Exercise Medicine 2021;7:e001215. doi:10.1136/ bmjsem-2021-001215

- Additional supplemental material is published online only. To view, please visit the journal online (http://dx.doi. org/10.1136/bmjsem-2021 001215).

Accepted 11 December 2021

\section{ABSTRACT}

Pain is often presumed to be part of the sport injury experience. The time-loss definition of injury leads to under-reported athletic pain impacting performance and quality of life. Whilst research regarding the assessment and classification of back pain in athletes is emerging, little has been reported regarding how peripheral pain is assessed and classified in research and practice. Six databases will be searched for relevant articles. Title and abstract screening followed by full-text screening will be completed by two independent reviewers. Data charting will be carried out using a modified standardised form. Descriptive results and frequencies will be reported. Pain measures identified in the studies will be mapped against the IOC Athlete Pain Framework alongside a narrative summary. Published peer-reviewed primary research studies alongside systematic reviews and clinical practice guidelines reporting the assessment or classification of pain in athletes of any age with chronic or acute peripheral pain across all study contexts in the English language on human participants from inception of the databases will be included. The results of this study are part of a body of research which will be used to inform the development of a pain assessment framework. The scoping review will be submitted for peer-reviewed journal publication and presented at sports medicine conferences. This review will inform researchers and clinicians working with athletes in pain how pain assessment and classification is currently conducted and positioned against the IOC Athlete Pain Framework.

\section{INTRODUCTION}

Pain is defined as 'an unpleasant sensory and emotional experience associated with, or resembling that associated with, actual or potential tissue damage' (Raja et al, p14). ${ }^{1}$ Pain is learnt through experience and is unique to each individual. ${ }^{1}$

Nociception, the way in which information regarding noxious stimuli is coded and interpreted by our nervous system, protects organisms from injury. ${ }^{2}$ Pain is generally adaptive, aiding in harm avoidance, but becomes maladaptive where pain persists despite the absence of harm. ${ }^{2}$ Contemporary pain understanding follows a holistic, interdisciplinary and multi-systems biopsychosocial framework. ${ }^{3} \quad$ Classifications

\section{Key messages}

What is already known

- Pain neuroscience and knowledge is advancing and a holistic biopsychosocial model is the gold standard to assess pain.

- The sports injury literature is advancing with improved knowledge and practice regarding the epidemiology, assessment, treatment and prevention of common sports injuries.

- Pain is part of the sports injury experience, however, focusing on sports injury alone may under-report athletic pain prevalence.

- In the last decade, research on the assessment and classification of athletic pain within a biopsychosocia framework has focused primarily on back pain.

\section{What are the new findings}

- This scoping review will explore the available literature to determine how peripheral pain is currently assessed and classified in athletes.

- Gaps for future research priorities in the assessment and classification of peripheral pain in athletes will be identified.

- Mapping the available evidence against the IOC Athlete Pain framework will inform researchers and clinicians managing athletes with peripheral pain of how current pain assessment and classification practice is positioned.

are based on pain mechanisms, presence of peripheral or central sensitisation, duration of symptoms and contribution of biopsychosocial factors. A comprehensive review of how the assessment and classification of peripheral pain is conducted has yet to be completed in athletic cohorts.

Describing athletes as solely elite competitive professionals has been challenged, with a definition of athletes based on the intent to compete, the level of competition and volume of weekly training being proposed. ${ }^{45}$ Using specific weekly training volume has also been debated due to potentially excluding athletes with lower training volume and including recreational exercisers with higher volumes. Hence, this study will define an athlete as anyone engaged in a sport, training regularly 
on a weekly basis, with the intent to improve performance and compete against others as an individual or as part of a team.

Athlete injury epidemiology is well established at both professional and amateur level across individual and team sports including athletics and rugby. ${ }^{78}$ The timeloss definition of injury regularly applied in the literature may under-report pain. ${ }^{9}$ A 1-year prevalence of athletic back pain of between $17 \%$ and $94 \%$ across a wide range of individual and team sports has been established. ${ }^{10}$ Similarly, peripheral pain (upper or lower limb) prevalence rates have been reported; hip and groin pain in footballers $(49 \%),{ }^{9}$ knee pain in volleyball players $(44.6 \%)^{11}$ and shoulder pain in swimmers $(38 \%-39.6 \%) .{ }^{12}$ Insidious onset and recurrent pain is common and often persists for greater than 6 weeks, impeding performance and negatively impacting function and quality of life. ${ }^{12}$

Stress and recovery, psychosocial factors and qualitative insights exploring the lived experience of pain reflect a shift in athletic back pain research and practice towards the biopsychosocial, neuromatrix and whole-person models currently implemented with general chronic low back pain populations. ${ }^{1314}$ To date research regarding the conduct of athletic pain assessment and classification has been focused primarily on back pain. Some general population psychosocial risk factor assessment tools have been applied successfully to athletes with thresholds being developed for identifying athletes at risk of going on to develop chronic low back pain. ${ }^{15}$ Improved understanding and assessment has paved the way for athletic back pain specific biopsychosocial management approaches (neuroscience education and cognitive functional therapy) to be developed. ${ }^{1617}$

Reporting guidelines, injury classification and coding systems have facilitated injury assessment models for a range of common peripheral sports injuries including ankle sprains and femoral acetabular impingement. ${ }^{18}{ }^{19}$ Specific treatment protocols, including those for the hamstring, adductor and calf and injury prevention programmes across sports including football and rugby have demonstrated efficacy. ${ }^{20}$ Psychosocial influences on injury risk, rehabilitation and return to sport have been well documented from the early stress-injury model to an integrative psychological, physiological, sociocultural and physical model. ${ }^{21}$ Despite extensive research on the topic of peripheral sports injury less has been reported regarding the specifics as to how athlete pain is and should be assessed, measured and classified in research and practice.

A review of the athletic pain assessment measures used in the Journal of Athletic Training (1992-1998) found that the majority of pain articles $(73.9 \% 17 / 23)$ focused on pain intensity through a simple pain scale with one third $(34.8 \% 8 / 23)$ using a secondary assessment tool that lacked validity. ${ }^{22}$ Over time, increases in the scope and breath of assessment encompassing pain, lifestyle, disability and psychological variables through the integration of pain rating scales, health-related quality of life, disability and function and psychological tools have been proposed..$^{23} 24$ These expert opinion based reviews, centred around clinical decision making for manual therapy, with no specific reference to peripheral pain, do not include any athlete specific pain assessment tools. The most recent International Olympic Committee consensus statement on pain management in elite athletes (IOC Athlete Pain Framework) and some related works providing athletic pain guidance in elite athlete cohorts highlight that an understanding of pain perception and the categories and mechanisms of pain is a priority for clinicians and researchers working with athletes. ${ }^{25-27}$ Currently little is reported about how peripheral pain has been assessed and classified in research and practice providing a lack of guidance for researchers, clinicians and indeed athletes. Pain assessment is an integral part of many research studies and clinical practice assessments. A gap exists for a comprehensive review of the pain assessment tools and classification strategies used across all research and practice settings.

This scoping review will examine the literature relating to the assessment and classification of athletic peripheral pain. It will highlight current practice for clinicians and map the pain assessment and classification tools against the IOC Athlete Pain Framework. In line with appropriate objectives for scoping reviews, it will also identify the gaps for future research. ${ }^{28}$

A preliminary search of PROSPERO, MEDLINE, the Cochrane Database of Systematic Reviews and the JBI Evidence Synthesis was conducted and no current or underway scoping reviews or systematic reviews on the topic of athlete peripheral pain assessment and classification were identified.

The objective of this scoping review is to establish the type of peripheral pain assessment and classification methods used with athletes in clinical, research and field settings.

\section{METHODS}

\section{Review question}

How is peripheral pain (sensory and emotional) assessed and classified in athletes?

\section{Aims}

To establish the sport (team/individual), anatomical site (upper/lower limb) and context (field/clinic/lab) of peripheral pain assessment and classification tools used in athlete cohorts.

To map the peripheral pain assessment tools highlighted in the review against the IOC Athlete Pain Framework.

To report the pain definitions and classifications used in athletic peripheral pain cohorts.

\section{Inclusion criteria}

\section{Participants}

Participants included in this review will be athletes of all ages who are currently engaged in a sport, training 
regularly on a weekly basis, with the intent to improve performance and compete against others either as an individual or as part of a team.

Recreational exercisers defined as anyone who participates in physical activity with the primary motivation to increase fitness, promote health, improve physique and learn or refine skills with an absence of intent to engage in competition against others will be excluded.

\section{Concept}

Acute or chronic pain originating in upper or lower limb regions (peripheral pain) will be included. Studies solely investigating pain originating in the spine or torso will be excluded. Peripheral pain must be assessed or classified. Studies reporting sensory measures of pain (eg, patient reported pain intensity scales, quantitative sensory testing and recording of pain location), emotional measures of pain (eg, patient reported pain related fear, anxiety and irritability) or a clearly defined pain classification system (eg, a biopsychosocial pain framework) will be included. Studies that focus solely on performance (including strength and movement assessments) or psychological (including personality, stress and mood) variables in the absence of pain measures will be excluded.

\section{Context}

All contexts of pain assessment measures will be included (preintervention and postintervention, outcome measures, diagnostic tools and research, clinical or field settings).

\section{Sources}

Published, peer-reviewed primary research with qualitative and/or quantitative data published in the English language on humans will be included. This includes epidemiological, questionnaire based, interview based, randomised and non-randomised controlled trials, prospective cohort, retrospective case-controls, crosssectional design and other primary study designs. Systematic reviews and clinical practice guidelines will be included only where primary assessment data is presented or reported. Given that primary data on pain assessment and classification tools used in research and practice are sought, review articles, editorial letters, book chapters, conference proceedings and websites along with other secondary research designs will be excluded.

\section{Methodology}

This scoping review will be conducted in accordance with the JBI methodology for scoping reviews. ${ }^{29}$ Building on a prior framework and methodological guidance, ${ }^{30} 31$ this approach facilitates enhanced development and reporting of appropriate objectives and comprehensive search strategies. ${ }^{29}$ This was achieved in the development of this protocol through a team based pre-planning phase to establish conceptual clarity alongside consultation with an information specialist/librarian to develop the search strategy. Conceptual clarity was established through the use of population, concept and context to focus the title, objectives and outcomes developing congruency, transparency and rigour beyond that proposed by earlier methodological frameworks. Furthermore this updated guidance is compatible with the latest Preferred Reporting Items for Systematic reviews and Meta-Analyses extension for Scoping Reviews (PRISMA-ScR) guidelines which will be used to report the results of this study. ${ }^{32}$

\section{Search strategy and sources of evidence selection}

The search strategy will aim to locate published primary studies, systematic reviews and clinical practice guidelines. An initial limited search of MEDLINE (PubMed) and CINAHL (EBSCO) was undertaken to identify articles on the topic. The text words contained in the titles and abstracts of relevant articles, and the index terms used to describe the articles were used to develop a full search strategy for MEDLINE (PubMed) (online supplemental appendix I). The search strategy, including all identified keywords and index terms, will be adapted for each database and searched from inception; CINAHL (EBSCOhost), MEDLINE (PubMed) PsychINFO (ProQuest), EMBASE (Elsevier), SCOPUS (Elsevier) and the Cochrane Database of Systematic Reviews. The reference lists of systematic review and clinical practice guidelines included in the review will be screened for additional papers. Studies will be limited to those published in English and on human subjects.

\section{Study selection}

Following the search, all identified records will be collated and uploaded into Endnote X9.3.3 (Clarivate Analytics, Pennsylvania, USA) and duplicates will be removed. Following a pilot test, titles and abstracts will then be screened by two independent reviewers for assessment against the inclusion and exclusion criteria for the review. Potentially relevant papers will be retrieved in full and their citation details imported into the Covidence reference management system (Covidence; Covidence Melbourne, Australia). The full-text of selected citations will be assessed in detail against the inclusion and exclusion criteria by two independent reviewers. Reasons for exclusion of full-text papers will be recorded and reported in the scoping review. Any disagreements that arise between the reviewers at each stage of the selection process will be resolved through discussion or with a third reviewer. The results of the search will be reported in full in the final scoping review and presented in a PRISMA-ScR flow diagram. ${ }^{32}$

\section{Data charting}

In line with methodological guidance, ${ }^{29}$ a pilot data charting exercise will be conducted independently by three members of the review team using a data charting tool adapted from the JBI Evidence Synthesis database. The data charted from studies will include study characteristics (citation details, country/region, research setting, study design, aims and limitations) participants' 
demographics, sport, competition level and region of pain. Specific measures relating to sensory and emotional aspects of pain as well as additional psychological and physical performance measures used will be charted. Pain definitions and classification systems used will be recorded. A specifically developed data charting tool (see online supplemental appendix II) will be modified and revised as necessary during the data charting process. Modifications will be detailed in the full scoping review. Authors of papers will be contacted to request missing or additional data, where required. If access to missing data is not possible these papers will be excluded.

\section{DATA ANALYSIS AND PRESENTATION}

Descriptive analysis of the range of assessment tools identified will be presented in table format. The authors will report the timelines/frequency for each pain assessment tool used, the study designs they were used in, the pain sites they were used for, along with all other IOC Athlete Pain Framework self-report cognitive, affective, sociocultural and objective physical performance measures collected alongside them.

Descriptive analysis with basic coding will be used for pain classification tools with the type and frequency of each classification type presented in table format. Frequencies of pain definitions will be reported.

The quantitative assessment measures used, and qualitative insights obtained will be coded and mapped visually against the categories contained within the IOC Athlete Pain Framework in line with suggested visual representation strategies. ${ }^{25} 28$

The IOC Consensus Framework comprises five domains. Neurophysiological (eg, establishing mechanism of pain, quantitative sensory testing and pain characteristics), biomechanical (eg, fatigue, training load, motor control and strength), cognitive (eg, attention, distraction, appraisal of pain and situation), affective (eg, mood, stress, fear and beliefs) and socioenvironmental (eg, social functioning, coach/parent responses, personal/ coach conflicts, pain culture and lifestyle) elements of pain. The framework further stipulates the need to identify and diagnose any specific pathology for underlying pain and the need to consider potential onward referral in a clinical setting. The pain assessment tools charted in this review will be coded to one or more of each of these five domains for all studies included. Frequencies of each tool per domain will be visualised and presented in figure format. As case report study designs are reports of clinical assessments, information on diagnosis of pathology and consideration of onward referral will be reported for these studies.

Through highlighting the current pain assessment tools used across the literature, specific domains within the IOC Athlete Pain Framework where pain assessment tools are and are not frequently used will be identified. This will allow future research to standardise pain assessment including all relevant fields thus allowing for comparison between research studies.
The findings of this review will be used to form the basis for a comprehensive peripheral pain assessment framework for athletes. The current IOC Athlete Pain framework provides an overview of the key domains of athletic pain assessment. There is a need to develop a practical framework that can be applied to elite and non-elite athletes that addresses specific pain assessment tools.

\section{DISCUSSION AND DISSEMINATION}

Whilst patient and public involvement has not been directly included in this study design, this study is part of a body of research which will seek patient and stakeholder consultation at the next phase (pain assessment framework development). The findings of this study will be discussed and communicated with patients (athletes) and clinicians (sports physiotherapists and physicians) prior to the development of an initial athletic peripheral pain assessment framework. Once published the results of the study will be summarised and shared in plain language to athletes in digital format (Twitter) and through the university sport clubs office via handouts. The findings will be shared with the Chartered Physiotherapists in Sports and Exercise Medicine (CPSEM) branch of the Irish Society for Chartered Physiotherapists through social media (Twitter) and at a national sports medicine study day to invite clinician feedback and discussion.

This study will establish how peripheral pain assessment is currently conducted. Mapping the available evidence against the IOC Athlete Pain Framework will inform researchers and clinicians managing athletes with peripheral pain of how current pain assessment and classification practice is positioned and identify specific domains where further emphasis is needed.

\section{Twitter Ciarán Purcell @Ciaran_Physio}

Acknowledgements Diarmuid Stokes, librarian in University College Dublin for his assistance with the search strategy. This review will contribute towards a PhD degree award from University College Dublin for the lead author.

Contributors $\mathrm{CP}$ and $\mathrm{BC}$ conceived the original idea. $\mathrm{CP}, \mathrm{BC}, \mathrm{CD}$ and $\mathrm{BF}$ developed the original idea. $\mathrm{CP}$ composed the initial manuscript draft. $\mathrm{BC}, \mathrm{CD}$ and $\mathrm{BF}$ provided comments on and contributed towards the writing and editing of the final draft.

Funding This work was supported by the Chartered Physiotherapists in Sports and Exercise Medicine (CPSEM) branch of the Irish Society of Chartered Physiotherapists through the yearly spring research bursary. This work was supported by funding from Science Foundation Ireland under the grant for the Insight SFI Research Centre for Data Analytics (SFI/12/RC/2289_P2).

Competing interests None declared.

Patient and public involvement Patients and/or the public were not involved in the design, or conduct, or reporting, or dissemination plans of this research.

Patient consent for publication Not applicable.

Ethics approval This study does not involve human participants.

Provenance and peer review Not commissioned; externally peer reviewed.

Data availability statement Data sharing not applicable as no datasets generated and/or analysed for this study. Not applicable.

Supplemental material This content has been supplied by the author(s). It has not been vetted by BMJ Publishing Group Limited (BMJ) and may not have been peer-reviewed. Any opinions or recommendations discussed are solely those 
of the author(s) and are not endorsed by BMJ. BMJ disclaims all liability and responsibility arising from any reliance placed on the content. Where the content includes any translated material, BMJ does not warrant the accuracy and reliability of the translations (including but not limited to local regulations, clinical guidelines, terminology, drug names and drug dosages), and is not responsible for any error and/or omissions arising from translation and adaptation or otherwise.

Open access This is an open access article distributed in accordance with the Creative Commons Attribution Non Commercial (CC BY-NC 4.0) license, which permits others to distribute, remix, adapt, build upon this work non-commercially, and license their derivative works on different terms, provided the original work is properly cited, appropriate credit is given, any changes made indicated, and the use is non-commercial. See: http://creativecommons.org/licenses/by-nc/4.0/.

\section{ORCID iD}

Ciarán Purcell http://orcid.org/0000-0002-4376-599X

\section{REFERENCES}

1 Raja SN, Carr DB, Cohen M, et al. The revised international association for the study of pain definition of pain: concepts, challenges, and compromises. Pain 2020;161:1976-82.

2 Baliki MN, Apkarian AV. Nociception, pain, negative Moods, and behavior selection. Neuron 2015;87:474-91.

3 Lin I, Wiles L, Waller R, et al. What does best practice care for musculoskeletal pain look like? eleven consistent recommendations from high-quality clinical practice guidelines: systematic review. $\mathrm{Br} \mathrm{J}$ Sports Med 2020;54:79-86.

4 Araújo CGS, Scharhag J. Athlete: a working definition for medical and health sciences research. Scand J Med Sci Sports 2016;26:4-7.

5 McKinney J, Velghe J, Fee J, et al. Defining athletes and Exercisers. Am J Cardiol 2019;123:532-5.

6 Maron BJ, Zipes DP, Kovacs RJ, et al. Eligibility and Disqualification recommendations for competitive athletes with cardiovascular abnormalities: Preamble, principles, and general considerations: a scientific statement from the American heart association and American College of cardiology. Circulation 2015;132:e256-61.

7 Fuller C, Taylor A, Douglas M, et al. Rugby world cup 2019 injury surveillance study. South African Journal of Sports Medicine 2020;32:1-6.

8 Edouard P, Navarro L, Branco P, et al. Injury frequency and characteristics (location, type, cause and severity) differed significantly among athletics ('track and field') disciplines during 14 international championships (2007-2018): implications for medical service planning. Br J Sports Med 2020;54:159-67.

9 Thorborg K, Rathleff MS, Petersen P, et al. Prevalence and severity of hip and groin pain in sub-elite male football: a crosssectional cohort study of 695 players. Scand J Med Sci Sports 2017;27:107-14.

10 Fett D, Trompeter K, Platen P. Back pain in elite sports: a crosssectional study on 1114 athletes. PLOS One 2017;12:e0180130.

11 Bahr R. No injuries, but plenty of pain? on the methodology for recording overuse symptoms in sports. Br J Sports Med 2009;43:966-72.

12 Oliveira VMAde, Pitangui ACR, Gomes MRA, et al. Shoulder pain in adolescent athletes: prevalence, associated factors and its influence on upper limb function. Braz J Phys Ther 2017;21:107-13.
13 Wilson F, Ng L, O'Sullivan K, et al. 'You're the best liar in the world': a grounded theory study of rowing athletes' experience of low back pain. Br J Sports Med 2021;55:327-35.

14 Fawcett L, Heneghan NR, James S, et al. Perceptions of low back pain in elite gymnastics: a multi-disciplinary qualitative focus group study. Phys Ther Sport 2020;44:33-40.

15 Wippert P-M, Puschmann A-K, Arampatzis A, et al. Diagnosis of psychosocial risk factors in prevention of low back pain in athletes (MiSpEx). BMJ Open Sport Exerc Med 2017;3:e000295.

16 Puentedura EJ, Louw A. A neuroscience approach to managing athletes with low back pain. Phys Ther Sport 2012;13:123-33.

17 Cañeiro JP, Ng L, Burnett A, et al. Cognitive functional therapy for the management of low back pain in an adolescent male rower: a case report. J Orthop Sports Phys Ther 2013;43:542-54.

18 Griffin DR, Dickenson EJ, O'Donnell J, et al. The Warwick agreement on femoroacetabular impingement syndrome (FAI syndrome): an international consensus statement. Br J Sports Med 2016;50:1169-76.

19 Orchard JW, Meeuwisse W, Derman W, et al. Sport medicine diagnostic coding system (SMDCS) and the orchard sports injury and illness classification system (OSIICS): revised 2020 consensus versions. Br J Sports Med 2020;54:397-401.

20 Ekstrand J, Spreco A, Bengtsson H. Injury rates decreased in men's professional football: an 18-year prospective cohort study of almost 12000 injuries sustained during 1.8 million hours of play. Br J Sports Med 2021.

21 Fisher H, Gittoes MJ, Evans L, et al. An interdisciplinary examination of stress and injury occurrence in athletes. Front Sports Act Living 2020;2:595619-19.

22 O'connor PJ, Murphy RM, Courson RW, et al. Pain assessment in Journal of athletic training articles 1992-1998: implications for improving research and practice. J Athl Train 2000;35:151-4.

23 Vela LI, Haladay DE, Denegar C. Clinical assessment of low-backpain treatment outcomes in athletes. J Sport Rehabil 2011;20:74-88.

24 Leznicka K. Evaluation of pain in athletes - Selected Methods. Central European Journal of Sport Sciences and Medicine 2013;3:27-30.

25 Hainline B, Derman W, Vernec A, et al. International Olympic Committee consensus statement on pain management in elite athletes. Br J Sports Med 2017;51:1245-58.

26 Zideman DA, Derman W, Hainline B, et al. Management of pain in elite athletes: identified gaps in knowledge and future research directions. Clin J Sport Med 2018;28:485-9.

27 Hainline B, Turner JA, Caneiro JP, et al. Pain in elite athletesneurophysiological, biomechanical and psychosocial considerations: a narrative review. Br J Sports Med 2017;51:1259-64.

28 Lockwood C, Dos Santos KB, Pap R. Practical guidance for knowledge synthesis: Scoping review methods. Asian Nurs Res 2019;13:287-94

29 Aromataris E, Z M, eds. JBM manual for evidence synthesis. Adelaide: JBI, 2020.

30 Arksey H, O'Malley L. Scoping studies: towards a methodological framework. Int J Soc Res Methodol 2005;8:19-32.

31 Levac D, Colquhoun H, O'Brien KK. Scoping studies: advancing the methodology. Implement Sci 2010;5:69.

32 Tricco AC, Lillie E, Zarin W, et al. PRISMA extension for scoping reviews (PRISMA-ScR): checklist and explanation. Ann Intern Med 2018;169:467-73. 